\title{
Partizipative Methoden zur Erfassung und Verarbeitung von Geoinformationen
}

Das Beispiel OpenStreetMap (OSM)

\author{
Luisa Griesbaum • Melanie Eckle • Benjamin Herfort • Martin Raifer • \\ Alexander Zipf $\mathbb{D}$
}

Eingegangen: 19. Februar 2017 / Angenommen: 19. Mai 2017 / Online publiziert: 24. Mai 2017 (C) Der/die Autor(en) 2017. Dieser Artikel ist eine Open-Access-Publikation.

Zusammenfassung Mit den steigenden Online-Partizipationsmöglichkeiten, die sich im Zuge des Web 2.0 seit geraumer Zeit ergeben, werden immer mehr Daten im Allgemeinen und Geodaten im Speziellen produziert. Durch Entwicklungen in GPS- und Satellitenbildtechnologie, können auch Laien ihre Umgebung und ferne Orte auf einfache Weise digital erfassen. Das wachsende Interesse an diesen Methoden zeigt sich in der Form von neuen digitalen Geodatensammlungen sowie webbasierten Werkzeugen zur Verarbeitung und Analyse dieser Daten.

OpenStreetMap (OSM) ist der bekannteste Vertreter von nutzer-generierten Geodaten. Diese werden auch Volunteered Geographic Information (VGI) genannt. Was 2004 mit dem Ziel begann, eine frei zugängliche Online-Straßenkarte zu entwickeln, ist inzwischen eine weltweite Geodatenbank für eine große Vielfalt an Geoobjekten. Die von Freiwilligen bereitgestellten OSM-Daten stehen unter Berücksichtigung der Open Database License (ODbL) jedem zur Verarbeitung, Nutzung und Verbreitung frei zur Verfügung. Somit ergeben sich immer mehr Anwendungen, sowie Möglichkeiten zur Partizipation. So entwickelte sich auch ein neuer Markt für Anwendungen,

L. Griesbaum $(\bowtie) \cdot$ M. Eckle $\cdot$ B. Herfort · M. Raifer · A. Zipf

Heidelberg Institute for Geoinformation Technology (HeiGIT), Abteilung

Geoinformatik/Geographisches Institut, Universität Heidelberg, Berliner Str. 45, 69120 Heidelberg,

Deutschland

E-Mail: griesbaum@uni-heidelberg.de

M. Eckle

E-Mail: eckle@uni-heidelberg.de

B. Herfort

E-Mail: herfort@uni-heidelberg.de

M. Raifer

E-Mail: martin.raifer@uni-heidelberg.de

A. Zipf
E-Mail: zipf@uni-heidelberg.de 
welche die Analyse und Informationsgewinnung aus eben diesen Daten unterstützen. $\mathrm{Zu}$ diesen zählen Routenplaner ebenso wie z. B. Karten zur Landnutzung. Die lange Liste der Anwendungen beinhaltet sowohl kommerzielle Angebote als auch gemeinnützige Aktivitäten.

Neben den Potentialen und Beteiligungsoptionen für Nutzer im Bereich von Geodaten und WebGIS, bilden auch die von den Freiwilligen generierten Daten und Herausforderungen v. a. bezüglich der großen Datenmengen und der Sicherstellung der Datenqualität einen zusätzlichen Forschungsgegenstand.

Schlüsselwörter OpenStreetMap · Volunteered Geographic Information · Routenplanung · Disaster Mapping

\section{Online Participation in Geoinformation Technology}

The example of OpenStreetMap (OSM)

Abstract In recent years, in the course of web 2.0 developments, online participation also reached the field of Geography, strictly speaking, the field of geo-information. More and more people start being engaged in collecting and disseminating geographic information about their local surroundings. This becomes apparent not only in forms of data collection, but also in form of data processing and the development of web based tools for data analysis.

OpenStreetMap (OSM) is one of the most popular examples of so-called Volunteered Geographic Information (VGI). While the initial objective of the project was the development of an open-access online street map, in the meantime the OSM database has become a profound source of information with high potential for participation far beyond a road map. Thus, nowadays even systematic and targeted mapping of all kinds of objects for various purposes, e. g. routing, land classification and disaster prevention, is conducted.

The OSM data is published under the Open Database License (ODbL) and therefore allows everyone to evaluate, analyze and process the OSM data. The emerging Web-GIS implementations comprise a wide range of use cases. These include commercial, as well as non-profit applications as for instance mapping and routing to support humanitarian aid and disaster management.

Apart from the research interest in the potentials in terms of geo-data availability and the enabling of participation, VGI and Web-GIS applications also lead to new research studies considering challenges and questions concerning (big) data quality and data handling.

Keywords OpenStreetMap · Volunteered Geographic Information · Routing · Disaster Mapping

\section{Einleitung}

Ein wesentliches Merkmal des Web 2.0 sind die von Nutzern bereitgestellten Inhalte auf Onlineplattformen. Hier werden Informationen und Daten aktiv erzeugt und mit 
anderen Nutzern online geteilt. Dadurch entwickelten sich zahlreiche partizipative Webseiten wie z. B. Wikipedia, sowie eine Reihe von Social Media Plattformen. Durch die verstärkte Verbreitung von mobilen Endgeräten können Nutzer auf diese Anwendungen auch mobil zugreifen und dadurch einfach selbst Daten aufnehmen und teilen. All diese nutzer-generierten Informationen werden als User-Generated Content (UGC) bezeichnet. Von zunehmenden Interesse sind Geodaten, Daten mit einem eindeutigen Raumbezug, welcher es erlaubt, die entsprechende Information auf der Erdoberfläche zu verorten.

Bei der Bereitstellung dieser Art von Geodaten wird zwischen implizit und expliziter Bereitstellung der Daten, sowie direkter und indirekter Geokodierung der Daten unterschieden. Sogenannte Volunteered Geographic Information (VGI) (Goodchild 2007) setzt den aktiven Beitrag von Freiwilligen voraus um entsprechende Informationen zu generieren oder zu sammeln. Dieser Praxis der zielgerichteten Datenerfassung steht die implizite Bereitstellung von geographischen Daten gegenüber, sogenannte Ambient Geographic Information (AGI). Diese Bezeichnung wird vor allem in Zusammenhang mit Social Media Daten verwendet, da diese Daten primär der Kommunikation mit anderen Nutzern dienen und nur zufällig (Geo-)Informationen produzieren. Neben der Einteilung nach Art der Erfassung und Geokodierung der Daten, können VGI-Projekte auch hinsichtlich des Art der Partizipation von Freiwilligen eingeteilt werden (Haklay 2013). Die Beteiligung kann von der Bereitstellung von Rechenkapazitäten über einfache Interpretationsaufgaben und Datenaufnahme bis hin zu extremer Beteiligung bezüglich Problembehandlungen und Projektgestaltungen reichen.

Nutzer-generierte Geodaten und partizipative Online-Tools, wie Werkzeuge zur Erfassung und Analyse der Daten, bereichern sich oft gegenseitig und zeigen viele weitere Potenziale. Daneben ist die freie Adaptierbarkeit der Daten als auch vieler Analysewerkzeuge für spezifische Anwendungen ein großer Gewinn. Durch das Mitwirken der Freiwilligen an den Programmen und Analysetools, können diese an deren Bedürfnisse angepasst werden (Haklay 2013). Diese neue Form der Datenerzeugung und vor allem die Nutzung von geographischen Techniken und Methoden von „Nichtgeographen“ wurde als Neo-Geographie bekannt.

Der folgende Beitrag soll einen Überblick über online Partizipationsmöglichkeiten und Potenziale im Bereich Geodaten-Erfassung, -Nutzung und -Verarbeitung geben. Welche nutzer-generierten Geodaten gibt es? Wie werden sie erfasst? Wie kann ich mich einbringen? Wozu können die Daten genutzt werden? Und wie steht es um die Qualität dieser Daten? Dazu wird zunächst eine Einführung die Strukturen und die Entwicklungen des prominentesten Vertreters von freien Geodaten, OpenStreetMap (OSM), gegeben, bevor im Weiteren ausgewählte Anwendungsmöglichkeiten dieser nutzer-generierten Geodaten aufgezeigt werden und die Herausforderungen hierbei diskutiert werden.

\section{Beispiel für online Partizipation - OpenStreetMap}

OSM stellt die bedeutendste Plattform zur Sammlung von nutzer-generierten Geodaten dar. Die Online-Weltkarte wurde 2004 mit dem Ziel gegründet eine freie Stra- 
Benkarte zu kreieren und wächst seitdem in beeindruckender Geschwindigkeit zu einer umfassenden, frei zugänglichen Geodatenbank heran. Neben den ursprünglich angedachten Wegenetzen und Objektdarstellungen sind inzwischen auch unzählige weitere Daten wie Adressen, Ladenöffnungszeiten, Straßenbeschaffenheit und weitere anwendungsspezifische Informationen in der OSM-Datenbank zu finden. Dies ist vor allem in der Vielfalt der OSM-Mitwirkenden und Nutzer begründet, sowie in der flexiblen Datenstruktur in OSM.

Jeder Nutzer kann je nach Interesse Daten in OSM editieren, ergänzen und verändern, frei nach dem Wiki-Prinzip. Durch die OSM-Gemeinschaft werden aber Richtlinien bereitgestellt und diskutiert, die vorgeben auf welche Weise bestimmte Objekte geometrisch abgebildet und klassifiziert (getagged) werden sollen. Die OSM-Datenstruktur besteht im Wesentlichen aus Punkten (X, Y-Koordinaten), welche beispielsweise die Position eines Baumes beschreiben. Mehrere aufeinander folgende Punkte können eine Linie (z. B. einen Straßenverlauf) darstellen und eine geschlossene Linie eine Fläche. Relationen beschreiben dabei Beziehungen zwischen verschiedenen Elementen oder Geometrien, wie etwa ein Gebäudeumriss mit einem Innenhof (in OSM beschrieben durch zwei geschlossene Linien). Tags sind Attribute, welche in OSM als eine Kombination aus Schlüssel und Wert vergeben werden. Beispielsweise wird durch den Tag highway=track einem Linienzug die Eigenschaft einer Straße zugewiesen. So lassen sich Objekttypen und ihre Eigenschaften beschreiben. Im OSM-Wiki sind die von der Community anerkannten Tags für die typischsten topographischen Objekte gesammelt ${ }^{1}$. Diese OSM-Richtlinien für Tags sind nicht verpflichtend, sondern es wird darauf vertraut, dass die Mitglieder untereinander durch die Überprüfung und Überarbeitung von gesammelten Daten und Informationen Fehler von Individuen ausgleichen. Getreu den VGI-Grundsätzen besteht generell die Annahme, dass eine größere Gruppe von Leuten aufgrund ihrer unterschiedlichen Kompetenzen ein Problem effizienter lösen kann als ein Individuum. Informationen, die von mehreren Personen gleichermaßen wahrgenommen werden, sollten demnach die Realität mit einer hohen Qualität abbilden.

Nutzer können im Rahmen des OSM-Projektes auf unterschiedliche Weise aktiv werden. Die Möglichkeiten reichen von Datenerhebung und -evaluation über die Entwicklung neuer Softwarewerkzeuge bis hin zur Teilnahme an speziellen Arbeitsgruppen, welche sich mit Fragen rund um die Organisation innerhalb der OSMCommunity beschäftigen. Die meisten Nutzer engagieren sich indem sie Daten erheben. Hierbei unterscheidet man zwischen zwei verschiedenen Ansätzen: local mapping und remote mapping. Im Fall des local mapping nehmen Nutzer ihre lokale Umgebung unter Zuhilfenahme von GPS-Gerät, Fotoapparat und/oder ausgedruckten, annotierbaren Papierkarten (sogenannten Fieldpapers) auf, um anschließend die gesammelten Informationen zu digitalisieren und in die OSM-Datenbank zu importieren. Das remote mapping hingegen erfordert nicht, dass die Freiwilligen direkt vor Ort sind, sondern ermöglicht das Digitalisieren von relevanten Objekten mithilfe von Luft- oder Satellitenbildern ${ }^{2}$. Letztere Methode ermöglicht auch ferne Orte, in denen unter Umständen weniger Zugang zu Technologie besteht, zu kartieren und

\footnotetext{
1 http://wiki.openstreetmap.org/wiki/DE:Map_Features.

2 http://wiki.openstreetmap.org/wiki/Armchair_mapping.
} 
auf die OSM-Karte zu bringen. Die so erhobenen Daten können in einem weiteren Schritt von lokalen Mappern mit zusätzlichen Attributen versehen werden.

Die OSM-Datenbank ist ein besonderes VGI-Beispiel, da nicht nur die Datenerhebung auf crowdsourcing basiert, also die Fähigkeiten eines Kollektivs genutzt werden um Informationen zu sammeln, sondern die Daten umgekehrt auch wieder der Allgemeinheit zur Verfügung gestellt werden und unter Berücksichtigung der Open Database License (ODbL) genutzt werden können ${ }^{3}$. Da die OSM-Datenbank durch die steigende Zahl der Mitwirkenden immer mehr an Detailgrad und Abdeckung gewinnt, werden OSM-Daten auch verstärkt in vielfältigen Anwendungen eingesetzt.

\section{Ausgewählte Anwendungsbereiche}

\subsection{Routenplanung und Navigation}

Da die OpenStreetMap mit dem Ziel eine freie weltweite Straßenkarte zu kreieren ins Leben gerufen wurde, ist es nicht überraschend, dass insbesondere Straßenund Wegeinformation mit hohem Detailgrad in der OSM-Datenbank erfasst sind. Folglich haben sich viele verschiedene Routing-Anwendungen entwickelt, welche auf Basis von OSM navigieren. Diese lassen sich in mobile (z. B. OSMand ${ }^{4}$ oder AndNav2 $2^{5}$ ) und browsergestützte Anwendungen (z. B. OpenRouteService ${ }^{6}$ ) unterscheiden. Der OpenRouteService (ORS) wurde als erster übernationaler Routenplaner auf OSM-Basis bereits ab 2007 entwickelt. Mittlerweile kann mit ORS weltweites Routing für verschiedene Transportmittel durchgeführt werden. Neben gängigen Funktionalitäten wie Fahrrad-, Fußgänger- und Auto-Routing mit zahlreichen Optionen erlaubt der ORS auch Truck- und E-Bike-Routing, sowie Routing für mobilitätseingeschränkte Menschen wie Rollstuhlfahrer (Zipf et al. 2016). Während bei dem Truck-Routing u. a. Straßenweite und -höhe relevant sind, spielen beim Routing für mobilitätseingeschränkte Personen vor allem die Oberflächenbeschaffenheit der Gehwege, deren Steigung und Verfügbarkeit von abgesenkten Bordsteinen eine Rolle, die individuell vom Anwender spezifiziert werden können. Gerade nutzergenerierte Plattformen wie OpenStreetMap ermöglichen es derartige spezielle Eigenschaften für Nutzer mit besonderen Informationsbedürfnissen vorzuhalten und können diesbezüglich flexibel erweitert werden. Als internationales Projekt besteht mit OSM erstmals die Möglichkeit über den Flickenteppich einzelner Kommunen und lokaler Initiativen hinweg eine gemeinsame überregionale Geodatenbasis auch zu solchen Spezialthemen zu realisieren, die dann auch überregional Routing und Navigation ermöglicht.

Auch können die gesammelten Daten genutzt werden um weitere Attribute abzuleiten, die nicht zwingend explizit erfasst werden. So liegen in den von lokalen

\footnotetext{
${ }^{3} \mathrm{http} / / /$ wiki.openstreetmap.org/wiki/Open_Database_License.

${ }^{4}$ http://osmand.net.

5 http://www.andnav.org.

${ }^{6}$ http://openrouteservice.org.
} 
Mappern hochgeladenen GPS-Tracks unter anderem auch Höhendaten vor. Diese können dazu genutzt werden Neigungen entlang den Strecken zu berechnen, die die Daten digitaler Geländemodelle ergänzen können (John et al. 2016). OpenRouteService nutzt Steilheitswerte nicht nur für Rollstuhlfahrer, sondern auch für spezielles eBike-Routing oder Fitness-spezifische Radroutingoptionen. Ein anderes Beispiel für die Ableitung neuer Eigenschaften aus den aufgenommenen Daten ist z. B. die Berechnung von Maßen für die Urbanität einer Region (Hagenauer und Helbich 2012), was wiederum für die Anpassung von Durchschnittsgeschwindigkeiten bei der Routenplanung genutzt werden kann.

Neben dem ORS gibt es mittlerweile weitere Routing-Anwendungen (z.B. Graphhopper ${ }^{7}$, Mapzen ${ }^{8}$, OSRM ${ }^{9}$ ), die OSM-Daten nutzen. Weitergehende Fragestellungen hierbei betreffen z. B. Evakuierungssimulationen (Outdoor und Indoor), Indoor-Routing, 3D-Routing, Verkehrsmanagement oder Routing auf Freiflächen wie großen Plätzen, die alle zunehmend OSM-Daten nutzen.

Als weitere Funktion unterstützt ORS sogenannte Erreichbarkeitsanalysen. Hierbei können jene Gebiete bestimmt werden, welche innerhalb einer bestimmten Zeit oder zu fahrenden Strecke erreichbar sind und dann als Isochronenkarte dargestellt werden (Abb. 1a). Dies ist Grundlage für weitere Anwendungen in Logistik, Planung und Verkehrsmanagement.

Eine spannende Nutzung stellt auch das Disaster-Routing dar. Im Katastrophenfall können vorliegende Basisdaten über Straßen von lokalen Mappern oder unter Zuhilfenahme aktueller Satellitenbilder mit spezifischen, gegebenenfalls temporären Informationen angereichert werden (siehe auch Abschn. 3.3). Dazu zählt beispielsweise die Kennzeichnung von eingestürzten Brücken oder unbefahrbaren Straßen. Diese zusätzlichen Informationen werden beim ORS Disaster-Routing berücksichtigt, sodass gesperrte Straßen oder Gebiete umfahren werden. Dies kam unter anderem schon bei Hilfseinsätzen bei Erdbeben in Haiti 2010, in Nepal 2016 und in Ecuador 2016 zum Einsatz.

Eine innovative Anwendung ist die Kombination von OpenStreetMap mit anderen nutzer-generierten Daten aus Web 2.0 Plattformen wie Flickr, Twitter etc., um gemäß wählbarer Kriterien für spezifische Nutzer ,interessante Routenvorschläge“ zu berechnen (Sun et al. 2015). Während Konzepte und Prototypen zur ,,personalisierten Routenplanung“" seit geraumer Zeit diskutiert werden, liegen in OSM sowie weiteren VGI und AGI Plattformen nun auch die für die Umsetzung notwendigen Daten vor. Dies gilt ähnlich für die sogenannte Landmarken-basierte Navigation (Rousell und Zipf 2017), die die Verständlichkeit von Routenbeschreibungen gegenüber herkömmlichen, v. a. metrischen Navigationsanweisungen nachweislich erhöhen (Richter und Winter 2014). All dies sind Beispiele dafür, welche neuen Potentiale Online-Partizipationsplattformen wie OSM eröffnen.

\footnotetext{
7 https://graphhopper.com/maps.

8 https://mapzen.com.

9 http://map.project-osrm.org.
} 
a

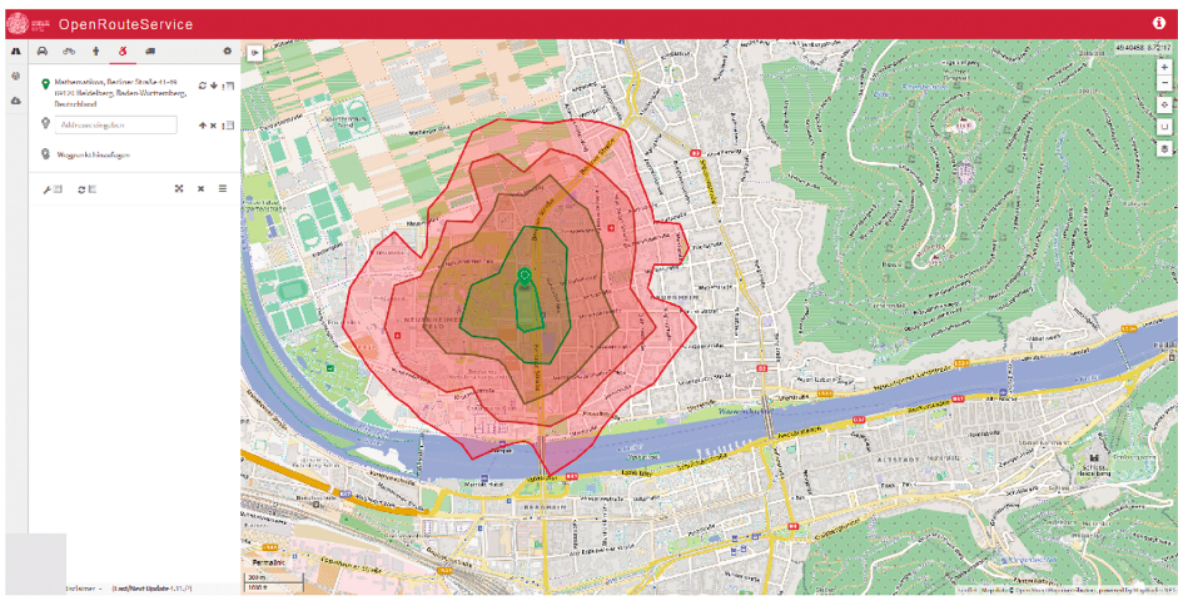

b

\section{Hist@̊SM}

a Infos Contact GIScience

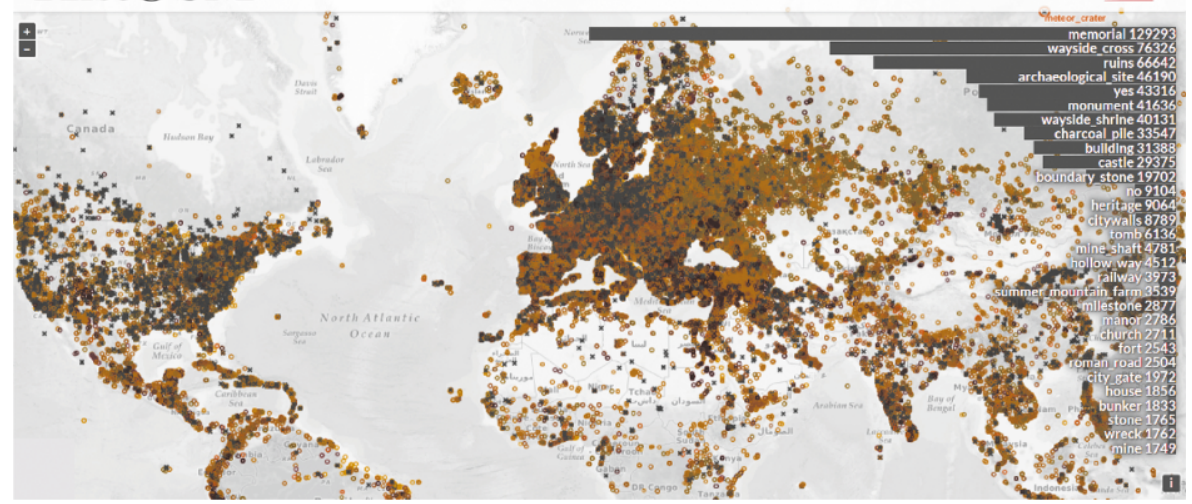

C

OSM

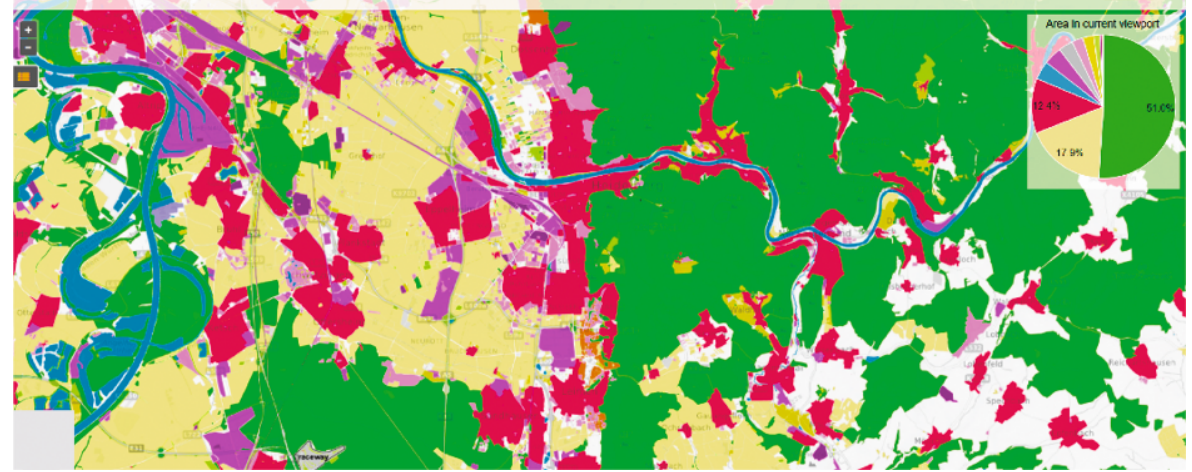

Abb. 1 Beispielanwendungen basierend auf OSM-Daten: a Erreichbarkeitsanalyse mit OpenRouteService, b HistOSM.org, c OSMlanduse.org 


\subsection{Thematische Kartenanwendungen}

Durch die Vielfalt an frei verfügbaren Daten in der OSM-Datenbank gibt es zahlreiche thematische Kartenanwendungen, welche die Daten angepasst an spezielle Zielgruppen und Anwendungsfälle visualisieren. Mit dem Wachsen der Community werden immer spezifischere Objekte in die OSM-Datenbank aufgenommen. Dazu gehören beispielsweise auch historische oder archäologische Objekte. So werden in der Anwendung HistOSM ${ }^{10}$ alle OSM-Objekte, die mit dem Schlüssel historic versehen sind, abgebildet und aktuelle Häufigkeitsstatistiken für die angezeigte Region eingeblendet (Auer et al. 2010) (Abb. 1b). Während solche Informationen bisher für Nicht-Experten eher weniger zugänglich in Fachbüchern zu finden waren, kann sich nun jedermann schnell einen Überblick über die geographische Verteilung des Kulturerbes einer Region verschaffen.

Ein ähnliches Prinzip wird in der OSM Landuse/Landcover Map ${ }^{11}$ verfolgt. Hier wird die Landnutzung in einer Region dargestellt. Dazu werden aus OSM entsprechende Flächen extrahiert und aufgrund ihrer zugehörigen Tags klassifiziert: Schlüssel, welche bestimmte Landnutzungstypen beschreiben, werden entsprechend des Klassifikationslevel 2 der CORINE Landnutzungsklassen zusammengefasst und kartographisch visualisiert. Zusätzlich wird eine Statistik über die Verteilung der ermittelten Landnutzungsklassen im dargestellten Gebiet angezeigt (Abb. 1c). Im Gegenzug zu z. B. CORINE-Daten der EU, welche in unregelmäßigen Abständen von mehreren Jahren neu erhoben werden, bietet die Grundlage von Landnutzungsklassen basierend auf OSM-Daten eine höhere Aktualität allerdings bei noch hoher räumlicher Heterogenität (Fonte et al. 2016).

Viele dieser thematischen Karten basieren auf einer OSM-Basiskarte. Durch das Hinzufügen weiterer Objektarten zur Basiskarte, können auf einfache Weise thematische Web-Karten erstellt werden. Dieses einfache Baukastensystem erlaubt auch Laien maßgeschneiderte Web-Anwendungen zu bauen. Rahmenwerke wie umap ${ }^{12}$ unterstützen diese Art der Online-Partizipation zur Verarbeitung, Analyse und Visualisierung von raumbezogenen Informationen.

\subsection{Katastrophenmanagement}

Ein besonderer Anwendungsfall für VGI und Web-GIS stellen humanitäre Katastrophen dar. In Katastrophensituationen oder humanitären Einsätzen sind Geodaten zur aktuellen Lage vor Ort von entscheidender Bedeutung um z. B. herauszufinden, welche Gebiete und Infrastrukturen besonders betroffen sind, wo sich potentielle Opfer und Gefahrenzonen befinden und, wie diese zu erreichen sind um weitere Planungen zum Einsatz und Logistik vornehmen zu können.

Die Einbindung von nutzer-generierten Geodaten hat sich in den letzten Jahren hierfür als sehr nützlich erwiesen. Dieses Potential wurde in der breiteren Öffentlichkeit erstmals nach dem schweren Erdbeben 2010 in Haiti bekannt. Hier bestand

\footnotetext{
10 https://HistOSM.org.

11 http://osmlanduse.org.

12 https://umap.openstreetmap.fr.
} 
a

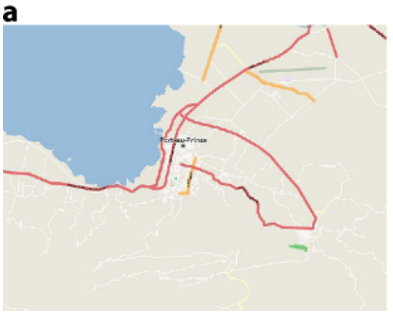

b

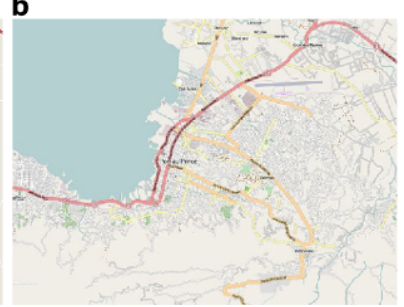

c

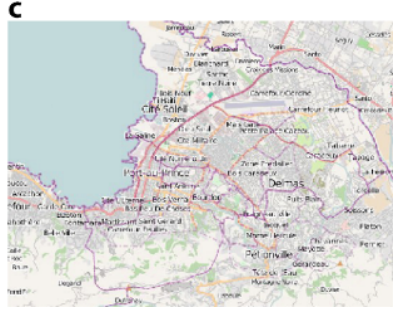

Abb. 2 OSM-Daten von Port-Au-Prince (Haiti): a vor dem Erdbeben Anfang 2010, b 2 Tage danach und c 2 Monate später

großer Bedarf an Informationen über die Lage vor Ort, die über traditionelle Wege nicht beschafft werden konnten. Um dieses Problem zu lösen, wurden von der weltweiten OSM-Gemeinschaft auf Grundlage von Satellitenbildern innerhalb kürzester Zeit detailreiche Karten in OSM erzeugt (Abb. 2). Aus diesen spontanen Hilfsaktionen hat sich unter anderem das Humanitarian OpenStreetMap Team (HOT $)^{13}$ gegründet. Dieses übernimmt die Koordination der OSM-Mapper, die Kommunikation mit den Hilfsorganisationen und Entwicklung von Werkzeugen, um die Erfassung und Nutzung der Daten im Katastrophenfall zu erleichtern. Seither hat es bei allen größeren Katastrophenereignissen, wie dem Tsunami in Japan (2011), Taifun Haiyan auf den Philippinen (2013), dem Erdbeben in Nepal (2015) eine Aktivierung der HOT-Gemeinschaft gegeben. Diese werden international durch Mapping Events sowie individuelle Mapper unterstützt. Schon früh wurden diese an der Universität Heidelberg durch die Entwicklung von Web-Diensten und Durchführung von „Mapathons“ hierbei vielfältig unterstützt (Reimer et al. 2014).

Beispielsweise wurde zur Unterstützung des lokalen Hochwassermanagements die OpenFloodRiskMap (OFRM) entwickelt, ein auf OSM-Daten basierendes Entscheidungsunterstützungssystem. Dieses erlaubt „Kritische Infrastrukturen“ zu identifizieren und durch die Integration von offiziellen Hochwassergefahrenkarten und des ORS unter Berücksichtigung von hochwasserbedingten Straßenverhältnissen zu diesen zu navigieren ${ }^{14}$ (Eckle et al. 2016). Durch die OSM-Datenbasis erlaubt die OFRM Entscheidungsträgern und Kommunen selbst an der Erhebung und Wartung der Daten mitzuwirken und die Datenqualität zu sichern. Zudem gibt es Projekte, die sich mit der Nutzung der OSM-Daten für die Katastrophenvorsorge und generelle humanitäre Hilfe befassen. Das 2014 gegründete Missing Maps Project ${ }^{15}$, ein Zusammenschluss des amerikanischen und britischen Roten Kreuzes, Ärzte ohne Grenzen UK und HOT, ist ein Beispiel dafür. Ziel dieses Projektes ist es, OSMDaten zur Unterstützung humanitärer Hilfseinsätze zu kreieren und bereits vor Katastrophenereignissen besonders vulnerable Gebiete zu kartieren. Durch die zusätzliche Kooperation mit Forschungs- und Bildungseinrichtungen wie der GIScience Research Group der Universität Heidelberg ${ }^{16}$ wurden in diesem Zuge auch neue

\footnotetext{
13 https://hotosm.org.

$14 \mathrm{http} / / /$ ofrm.geog.uni-heidelberg.de/map.

$15 \mathrm{http}: / /$ missingmaps.org.

16 http://www.geog.uni-heidelberg.de/gis/index_en.html.
} 
Verfahren zur Unterstützung des Kartierens für humanitäre Einsätze entwickelt. Unter anderem wurde im Laufe der Kooperation ein neues Web-basiertes Verfahren zur Vereinfachung der Datenaufnahme entwickelt. Die, zunächst browsergestützte, Anwendung zur Klassifizierung von Satellitenbildern in bewohnte und unbewohnte Gebiete wurde inzwischen in eine mobile Version überführt (MapSwipe App). MapSwipe ${ }^{17}$ ermöglicht auch nicht OSM affinen Freiwilligen durch einfaches Tippen auf dem Smartphone Informationen über besiedelte Gebiete zu erheben, die im weiteren Schritt das Mappen dieser Regionen erleichtern (Porto de Albuquerque et al. 2016). Die Einfachheit dieser Anwendung ermöglicht ganz neuen breiten Zielgruppen an Freiwilligen ohne spezielle Hintergrundkenntnisse bei der Unterstützung humanitärer Aktionen im OSM-Kontext zu partizipieren.

\section{Herausforderungen von VGI-Daten}

Neben den vielen Potentialen von VGI, gibt es auch diverse Schwierigkeiten, die sich durch die Art der Datenerhebung ergeben. Eine der größten Herausforderungen stellt hierbei die schwankende Vollständigkeit und Qualität der Daten dar. Da VGI-Projekte auf Beiträge der Projektmitglieder angewiesen sind, sind diese auch ausschlaggebend für die Abdeckung, den Detailgrad und die Qualität der Daten. Dies kann am Beispiel von OSM veranschaulicht werden. So zeigt unter anderem die Untersuchung von Neis et al. (2013), dass die Vollständigkeit der OSMDaten regional stark variiert. So ist die Abdeckung typischerweise in städtischen Gebieten und Ballungsräumen umfassender als in ländlichen Gebieten. Neben der Vollständigkeit spielt zudem die Konsistenz des Taggings von Objekten in OSM eine bedeutende Rolle. Da es in OSM keine verpflichtenden Daten-Standards gibt, sondern nur von der OSM-Gemeinschaft festgelegte Richtlinien, wie Informationen in der OSM-Datenbank abzubilden und zu kennzeichnen sind (Folksonomie), kann generell jeder Nutzer Daten auf seine eigene Art und Weise eingeben. Hier setzen auch Überlegungen zur konzeptionellen Qualität von VGI Daten an (Ballatore und Zipf 2015).

In den letzten Jahren sind zahlreiche Forschungsarbeiten veröffentlicht worden, welche sich mit der Qualität von VGI beschäftigen. Neben Vergleichen mit amtlichen oder kommerziellen Datensätzen, steht auch die intrinsische Datenqualitätsanalyse im Fokus (Barron et al. 2014). Sie erlaubt eine Abschätzung der Datenqualität aufgrund der Daten selbst inhärenten Eigenschaften. Unter anderem werden hierbei die Art der Datenerhebung betrachtet, sowie die Aktivitäten der Erfasser (z. B. Jokar Arsanjani et al. 2013). Beispiele für entsprechende Analysewerkzeuge und Dienste sind iOSMAnalyzer oder OSMatrix ${ }^{18}$ (Roick et al. 2012). Der iOSMAnalyzer ermittelt verschiedene Qualitätsparameter durch Untersuchung der Bearbeitungshistorie der OSM-Daten eines bestimmten Gebiets. Hierbei wird die Veränderung der Daten über die Zeit betrachtet und Aspekte, wie etwa die Vollständigkeit der Attributierung, untersucht (Barron et al. 2014). OSMatrix erlaubt derartige Qualitätsparameter

\footnotetext{
17 http://mapswipe.org.

18 http://osmatrix.uni-hd.de.
} 
auf einer Karte zu visualisieren. Dazu gehören Parameter wie die Anzahl der OSMMitglieder, die in der jeweiligen Region aktiv waren oder das (Nicht-)Vorhandensein bestimmter Objektarten. Relevant ist zudem die Frage nach Vandalismus in nutzergenerierten Geodaten.

Neuste Untersuchungen befassen sich mit der Anwendung von Machine-Learning Verfahren zur Datenqualitätsanalyse und Verbesserung. Ein weiteres Ziel aktueller Forschung ist es, die Eigenschaften der OSM-Daten sowie die zugrundeliegenden Satellitendaten über Machine Learning miteinander in Verbindung zu setzen.

\section{Fazit/Ausblick}

Die Anwendungs- und Analysemöglichkeiten von UGC, insbesondere VGI, sind vielfältig. Diese reichen von Themen wie Navigation über die Visualisierung und Analyse von speziellen Themenbereichen in Form von teils interaktiven OnlineKarten bis hin zur Nutzung der Daten in humanitären Hilfseinsätzen. Für all diese Anwendungsbereiche gibt es unterschiedliche Ebenen der Mitwirkung: Die Daten werden zum einen erhoben, evaluiert, analysiert und visualisiert, und zum anderen immer weitere Methoden und Werkzeuge entwickelt, um genau diese Aufgaben zu erleichtern.

Speziell für das Katastrophenmanagement werden Anwendungen entwickelt, welche diese neuen Datenquellen effektiv nutzen, um das Lagebild und die Arbeit der Hilfsorganisationen zu verbessern. Dazu zählt die Datenintegration der UGC in offizielle Datenstrukturen oder die schnelle Auswertung der Daten.

Ebenso ergeben sich Fragen nach der Qualität der Daten von OSM als aktuelles Forschungsfeld. Dabei geht es um die Analyse, Bewertung und Verbesserung der Datenqualität. Diese werden auf Genauigkeit, Vollständigkeit, Aktualität, Konsistenz in Geometrie und Semantik untersucht, um die Tauglichkeit der Daten für die verschiedenen Anwendungsfelder einschätzen zu können. Einen Überblick über weitere Forschungsaspekte in Bezug auf VGI und OSM geben Bücher wie Jokar Arsanjani et al. (2015) oder allgemeiner Sui et al. (2013).

Open Access Dieser Artikel wird unter der Creative Commons Namensnennung 4.0 International Lizenz (http://creativecommons.org/licenses/by/4.0/deed.de) veröffentlicht, welche die Nutzung, Vervielfältigung, Bearbeitung, Verbreitung und Wiedergabe in jeglichem Medium und Format erlaubt, sofern Sie den/die ursprünglichen Autor(en) und die Quelle ordnungsgemäß nennen, einen Link zur Creative Commons Lizenz beifügen und angeben, ob Änderungen vorgenommen wurden.

\section{Literatur}

Auer M, Fees M, Zipf A (2010) HistOSM.org - ein Webportal zu historischen und archäologischen Stätten und Sehenswürdigkeiten auf Basis der nutzergenierten Daten von OpenStreetMap (OSM). AGIT 2010 Symposium für Angewandte Geoinformatik, Salzburg.

Ballatore A, Zipf A (2015) A conceptual quality framework for volunteered geographic information. COSIT 2015 Conference on Spatial Information Theory XII, Santa Fe.

Barron C, Neis P, Zipf A (2014) A comprehensive framework for intrinsic openstreetmap quality analysis. Trans Gis 18(6):877-895. doi:10.1111/tgis.12073 
Eckle M, Porto de Albuquerque J, Herfort B, Zipf A (2016) Freie Geodaten zur Entscheidungsstützung im Hochwassermanagement - Fallbeispiel OpenFloodRiskMap. AGIT 2016 Symposium für Angewandte Geoinformatik, Salzburg.

Fonte CC, Minighini M, Antoniou V, See L, Patriarca J, Brovelli MA, Milcinski G (2016) Automated methodology for converting OSM data into a land use/cover map. In: Proceedings of the 6th International Conference on Cartography \& GIS Albena. Bd. 1, S 462-473

Goodchild MF (2007) Citizens as sensors: the world of volunteered geography. GeoJournal 69(4):211-221. doi:10.1007/s10708-007-9111-y

Hagenauer J, Helbich M (2012) Mining urban land-use patterns from volunteered geographic information by means of genetic algorithms and artificial neural networks. Int J Geogr Inf Sci 26(6):963-982. doi:10.1080/13658816.2011.619501

Haklay M (2013) Citizen science and volunteered geographic information: overview and typology of participation. In: Sui D, Elwood S, Goodchild M (Hrsg) Crowdsourcing geographic knowledge. Springer, Dordrecht, S 105-122

John S, Hahmann S, Rousell A, Löwner M-O, Zipf A (2016) Deriving incline values for street networks from voluntarily collected GPS traces. Cartogr Geogr Inf Sci 44(2):152-169. doi:10.1080/15230406. 2016.1190300

Jokar Arsanjani J, Barron C, Bakillah M, Helbich M (2013) Assessing the quality of openstreetmap contributors together with their contributions. In: Vandenbroucke D (Hrsg) Proceedings of the 16th AGILE conference. Springer, Leuven

Jokar Arsanjani J, Zipf A, Mooney P, Helbich M (2015) Openstreetmap in GIScience: experiences, research, and applications. Springer International, Cham

Neis P, Zielstra D, Zipf A (2013) Comparison of volunteered geographic information data contributions and community development for selected world regions. Future Internet 5(2):282-300. doi:10.3390/ fi5020282

Porto de Albuquerque J, Herfort B, Eckle M (2016) The tasks of the crowd: a typology of tasks in geographic information crowdsourcing and a case study in humanitarian mapping. Remote Sens (Basel) 8(10):859. doi:10.3390/RS8100859

Reimer A, Neis P, Rylov M, Schellhorn S, Sagl G, Resch B, Porto de Albuquerque J, Zipf A (2014) Erfahrungsbericht: Crisis Mapping zum Taifun Hayan. In: Seyfert E, Gülch E, Heipke C, Schiewe J, Sester M (Hrsg) Geoinformationen öffnen das Tor zur Welt. DGPF Tagungsband 23/2014

Richter K-F, Winter S (2014) Landmarks; GIScience for intelligent services. Springer International, Cham

Roick O, Loos L, Zipf A (2012) A technical framework for visualizing spatio-temporal quality metrics of volunteered geographic information. In: Proceedings of the GEOINFORMATIK 2012 - mobility and environment Braunschweig. Shaker, Germany

Rousell A, Zipf A (2017) Towards a landmark-based pedestrian navigation service using OSM data. ISPRS Int J Geoinf 6(3):64. doi:10.3390/ijgi6030064

Sui D, Elwood S, Goodchild M (2013) Crowdsourcing geographic knowledge: volunteered geographic information (VGI) in theory and practice. Springer, Dordrecht

Sun Y, Fan H, Bakillah M, Zipf A (2015) Road-based travel recommendation using geo-tagged images. Comput Environ Urban Syst 53:110-122. doi:10.1016/j.compenvurbsys.2013.07.006

Zipf A, Mobasheri A, Rousell A, Hahmann S (2016) Crowdsourcing for individual needs - the case of routing and navigation for mobility-impaired persons. In: Capineri C, Haklay M, Huang H, Antoniou V, Kettunen J, Ostermann F, Puves R (Hrsg) European handbook of crowdsourced geographic information. Ubiquity, London, S 325-337 